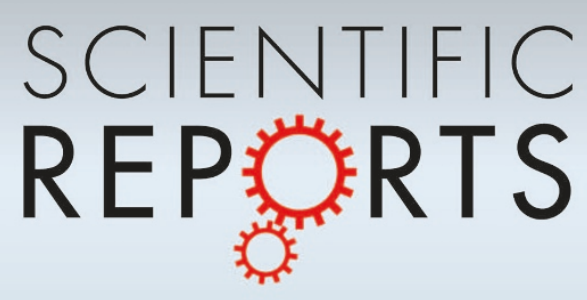

OPEN

SUBJECT AREAS:

PLANT STRESS

RESPONSES

METABOLOMICS

ECOTOXICOLOGY

ENVIRONMENTAL CHEMISTRY

Received

13 May 2014

Accepted

30 July 2014

Published

19 August 2014

Correspondence and requests for materials should be addressed to Q.Z. (zhouqx@nankai. edu.cn)

\section{Graphene oxide amplifies the phytotoxicity of arsenic in wheat}

\author{
Xiangang Hu', Jia Kang', Kaicheng Lu', Ruiren Zhou', Li Mu³ \& Qixing Zhou' \\ ${ }^{1}$ Key Laboratory of Pollution Processes and Environmental Criteria (Ministry of Education), Tianjin Key Laboratory of Environmental \\ Remediation and Pollution Control, College of Environmental Science and Engineering, Nankai University, Tianjin 300071, China, \\ ${ }^{2}$ College of Life Science, Nankai University, Tianjin 300071, China, ${ }^{3}$ Institute of Agro-environmental Protection, Ministry of \\ Agriculture, Tianjin 300191, China.
}

Graphene oxide (GO) is widely used in various fields and is considered to be relatively biocompatible. Herein, "indirect" nanotoxicity is first defined as toxic amplification of toxicants or pollutants by nanomaterials. This work revealed that GO greatly amplifies the phytotoxicity of arsenic (As), a widespread contaminant, in wheat, for example, causing a decrease in biomass and root numbers and increasing oxidative stress, which are thought to be regulated by its metabolisms. Compared with As or GO alone, GO combined with As inhibited the metabolism of carbohydrates, enhanced amino acid and secondary metabolism and disrupted fatty acid metabolism and the urea cycle. GO also triggered damage to cellular structures and electrolyte leakage and enhanced the uptake of GO and As. Co-transport of GO-loading As and transformation of As(V) to high-toxicity As(III) by GO were observed. The generation of dimethylarsinate, produced from the detoxification of inorganic As, was inhibited by GO in plants. GO also regulated phosphate transporter gene expression and arsenate reductase activity to influence the uptake and transformation of As, respectively. Moreover, the above effects of GO were concentration dependent. Given the widespread exposure to As in agriculture, the indirect nanotoxicity of GO should be carefully considered in food safety.

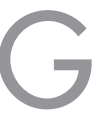
raphene oxide (GO) nanosheets, a novel engineered nanomaterial, have been applied in various fields, such as biology, chemistry, medicine, exploration and environmental protection ${ }^{1,2}$. Before using GO in such applications, it is essential to scientifically understand ecological risk and health significance of this material. The interactions of GO with biological molecules (DNA and proteins) as well as its cytotoxicity, uptake, pharmacokinetics and hematopathology have mainly been examined in animal experiments ${ }^{3,4}$, while its phytotoxicity remains largely unexplored. Considering that plants are the primary producers in ecosystems, understanding the interactions between nanomaterials and plants is crucial for comprehending the impact of nanomaterials on ecosystems and food safety. However, little progress has been made in this arena ${ }^{5-7}$.

The direct nanotoxicity of GO observed in living organisms suggests that GO is relatively biocompatible. For instance, GO acts as a general enhancer of cellular growth by increasing cell attachment and proliferation ${ }^{8}$. Further investigations based on histological examination of organ slices and hematological analyses revealed that GO toxicity in the treated animals was insignificant ${ }^{9}$. The positive impacts of $400 \mathrm{mg} / \mathrm{L}$ of GO included significant improvement of the plant health status indicated by decreased levels of $\mathrm{H}_{2} \mathrm{O}_{2}$ and lipid and protein oxidation $^{10}$. In fact, exposure of living organisms to multi-toxicants or pollutants is generally concomitant in the natural environment, rather than occurring in single exposure events. For instance, multiwalled carbon nanotubes and fullerenes increase the accumulation of pesticides in agricultural plants, with implications for food safety ${ }^{11}$. Fullerenes also increase the toxicity and bioaccumulation of xenobiotic organic compounds in algae and crustaceans $^{12}$. Diuron sorbed onto carbon nanotubes enhances toxicity to Chlorella vulgaris ${ }^{13}$. Nano- $\mathrm{Fe}_{2} \mathrm{O}_{3} / \mathrm{Al}_{2} \mathrm{O}_{3}$ significantly enhances the toxicity and accumulation of arsenic (As) (V) compounds in Ceriodaphnia dubia ${ }^{14}$. These results all indicate toxic amplification of toxicants or pollutants by nanomaterials. Herein, this phenomenon is defined as "indirect" nanotoxicity.

In addition to the amplification of uptake indicated above, "indirect" nanotoxicity can involve multiple phenomena, such as toxic amplification of coexistent toxicants via the regulation of transformation, metabolism, genes, proteins and enzymes. As is a ubiquitous toxic element and shows the potential to pose a great risk to animal and human health ${ }^{15}$. GO, which has a large hydrophilic-specific surface, can directly adsorb and indirectly transform As species ${ }^{16-18}$. Wheat (Triticum aestivum L.) is one of the most important food crops globally and is a recommended model organism in the guidelines for chemical testing proposed by the Organization for Economic 
Co-operation and Development (OECD) $)^{19}$. Widespread As contamination in wheat is a global issue $e^{20-22}$. The influence of GO on the uptake, transformation, metabolism and molecular toxicity of As in wheat is a critical issue in food safety. However, there is little available information related to this topic.

The primary goals of this study were to thoroughly assess the indirect nanotoxicity of GO in relation to As in wheat. Specifically, we determined: (i) the effects of GO on phytotoxicity of As, including growth inhibition and oxidative stress; (ii) the metabolic regulation of toxicity amplification; (iii) the influence of cell structure damage and permeability on the uptake of GO and As; (iv) the regulation of As uptake and transformation through the chemical interactions of GO; and (v) the regulation of As uptake and transformation through the biological interactions of GO, such as through gene expression and enzymes catalysis.

\section{Results}

Characteristics of GO. The surface chemistry of GO is described in Figure S1. X-ray photoelectron spectroscopy (XPS) images showed that GO included $59.99 \% \mathrm{C} 1 \mathrm{~s}, 36.57 \% \mathrm{O} 1 \mathrm{~s}$ and $3.44 \% \mathrm{~N} 1 \mathrm{~s}$. C1s was shown to be a composite of C-C (46.79\%), C-H (42.59\%) and C-O-C $(10.62 \%)$, while $\mathrm{O} 1 \mathrm{~s}$ was a composite of $\mathrm{C}=\mathrm{O}(75.24 \%)$ and $\mathrm{O}=\mathrm{C}-\mathrm{O}$ $(24.76 \%)$ and $\mathrm{N} 1 \mathrm{~s}$ was attributed to $\mathrm{N}-\mathrm{C}(88.20 \%)$ and $\mathrm{N}=\mathrm{C}$ $(11.80 \%)$. These oxygen- and nitrogen-containing groups result in the hydrophilic surface of GO. The characteristics of GO are further analyzed in Figure S2. A peak at $230 \mathrm{~nm}$ and a peak shoulder from approximately 280 to $300 \mathrm{~nm}$, which are the typical adsorption peaks of GO, were observed. There was no obvious alteration of spectra after 8 days in the GO $(10 \mathrm{mg} / \mathrm{L})$ solution, suggesting that GO is very stable in the aqueous phase. This excellent stability represents a benefit for the interactions between GO and organisms. Field emission transmission electron microscopy images showed irregular folds on GO nanosheets, which reflected the flexibility of GO. Atomic force microscope imaging demonstrated that the thickness of GO nanosheets was approximately $0.8-1 \mathrm{~nm}$. The diameter ranged from approximately $1-5 \mu \mathrm{m}$. Hence, as a wide size distribution would increase the nondeterminacy of the nanotoxicity test GO was treated using ultrasound $(150 \mathrm{~W}$, $30 \mathrm{~min}$ ) prior to nanotoxicity testing, leading to a narrow size distribution ( $465-486 \mathrm{~nm}$, centered on $476 \mathrm{~nm}$ ). The Raman spectrum was used to characterize the structure of GO, as shown in Figure S3. The typical D and G bands of graphene, located at approximately 1355 and $1602 \mathrm{~cm}^{-1}$, were detected; these bands reflect the disordered structure and ordered $s p^{2}$ carbon system of graphene, respectively ${ }^{23,24}$. Other features included the $2 \mathrm{D}$ band and combined-mode D + G band located at approximately 2747 and $2939 \mathrm{~cm}^{-1}$, respectively. The positions and intensity of the 2D bands reflect the number of graphene layers ${ }^{23,24}$ : the $2 \mathrm{D}$ band of single-layer graphene centers around $2679 \mathrm{~cm}^{-1}$, and the $2 \mathrm{D} / \mathrm{G}$ intensity ratio of single-layer graphene is $>1.6^{24}$. In this work, the 2D peak was located at approximately $2747 \mathrm{~cm}^{-1}$, and the $2 \mathrm{D} / \mathrm{G}$ intensity ratio was found to be 0.6 for the single-layer GO. Compared to pristine graphene, the disagreement likely was due to the oxygen-containing groups in $\mathrm{GO}^{25}$.

GO amplifies the toxicity of $\mathbf{A s}(\mathrm{V})$. The effects of GO and $\mathrm{As}(\mathrm{V})$ on wheat seed germination are presented in Figure 1. Compared with the control, As(V) and GO did not significantly inhibit seed germination, by having assessed using the fresh weight, the germination rate, the root length, the root number and the shoot length, as shown in Figure 1a. Concerning positive effects, As(V) slightly increased the germination rate and the root number. The shoot length significantly reduced with an increase in the GO concentration in the AsGO groups. It appears that GO directly affected the shoot length and $\mathrm{As}(\mathrm{V})$ enhanced the impact of GO under the condition of $\mathrm{As}(\mathrm{V})$ and GO co-exposure. AsGO 0.1 markedly $(P<0.05)$ reduced the shoot length and this reduction was more intensive with an increase in GO concentrations. AsGO 10 significantly reduced both the fresh weigh and the shoot length. Chlorophyll biosynthesis, malondialdehyde (MDA) contents and the activities of peroxidase (POD) and superoxide dismutase (SOD) exhibited similar effects to growth inhibition, as shown in Figure $1 b$. There were no significant differences between the control and As(V) groups. GO directly affected the SOD and POD activities, and the toxicity was significantly amplified by the coexposure of $\mathrm{As}(\mathrm{V})$ and GO. AsGO induced a significant reduction of chlorophyll contents, especially that of chlorophyll b. The activity of SOD increased with an increase in the concentration of GO in the AsGO groups. The changes in MDA as an indicator of lipid peroxidation were not significant among all groups. The above results suggested that the relatively biocompatible GO markedly enhanced the phytotoxicity of As.

Metabolism regulates the amplification of nanotoxicity. Compared with proteins and genes, metabolic changes directly reflect biological processes ${ }^{26,27}$. Herein, metabolomics analyses were conducted to elucidate the mechanisms of indirect nanotoxicity of GO in the presence of As. A total of 200 peaks in each sample were analyzed, and 65 metabolites were identified, as shown in Tables S1 and S2. Compared with other results from metabolic analyses (e.g., using ${ }^{1} \mathrm{H}$ NMR technology), the applied extraction procedure and gas chromatography-mass spectrometry/mass spectrometry with the derivatization method were effective for analyzing the multiclass metabolites of plant cells ${ }^{28,29}$. These metabolites correspond to the main metabolic pathways involved in carbohydrate, amino acid and fatty acid production, secondary metabolism and the urea cycle. The metabolites were analyzed using t-tests in Multiple Experiment Viewer 4, and the metabolites that were found to show significant differences $(P<0.05)$ in the roots and shoots, which are listed in Figure $2 \mathrm{a}$ and $2 \mathrm{~b}$, respectively. Based on hierarchical clustering (HCL) analysis, the root metabolites were divided into two clusters: the control and As/GO/AsGO. Furthermore, the As/GO/ AsGO cluster was divided into two sub-clusters: As and GO/AsGO. Similarly, the shoot metabolites were divided into two clusters: the control and As/GO/AsGO; and As/GO/AsGO was further divided into two sub-clusters: As/GO and AsGO. Principal component analysis (PCA) was also employed to illustrate the metabolic specificity among different groups (Figure 2c). The samples were divided into two groups: the control and As/GO/AsGO. The discrete scores suggested that the metabolism displayed significant differences among the As, GO and AsGO groups. The above results demonstrated that AsGO markedly altered the metabolism in the control, As and GO groups.

The results in Figure 1 suggest that the root number and chlorophyll b content were sensitive to AsGO toxicity in the roots and shoots, respectively. To further assess metabolic regulation, using both the root number and chlorophyll $b$ as $Y$ variables, an orthogonal partial least squares discriminant analysis (OPLS-DA) model was implemented (Figures S4-6). In the roots, 7 of 23 metabolites showed a positive coefficient CS (coeffCS) value, indicating that these metabolites made positive contributions to the observed root number. Among these 7 metabolites, AsGO reduced the contents of xylose, maltose, aspartic acid and aconitic acid with a VIP (variable importance for the projection) of more than 0.7. Accordingly, the remaining metabolites made negative contributions to the observed root number. AsGO increased the contents of 16 metabolites, and the metabolites exhibiting a VIP of more than 1.4 included naphthalene, monopalmitin, asparagine, galactose and cyclononasiloxane octadecamethyl-. In the shoots, 9 of 57 metabolites with a positive coeffCS made positive contributions to the anabolism of chlorophyll $b$. Among these 9 metabolites, AsGO decreased the contents of galactopyranoside and galactose, which showed a positive coeffCS with a 

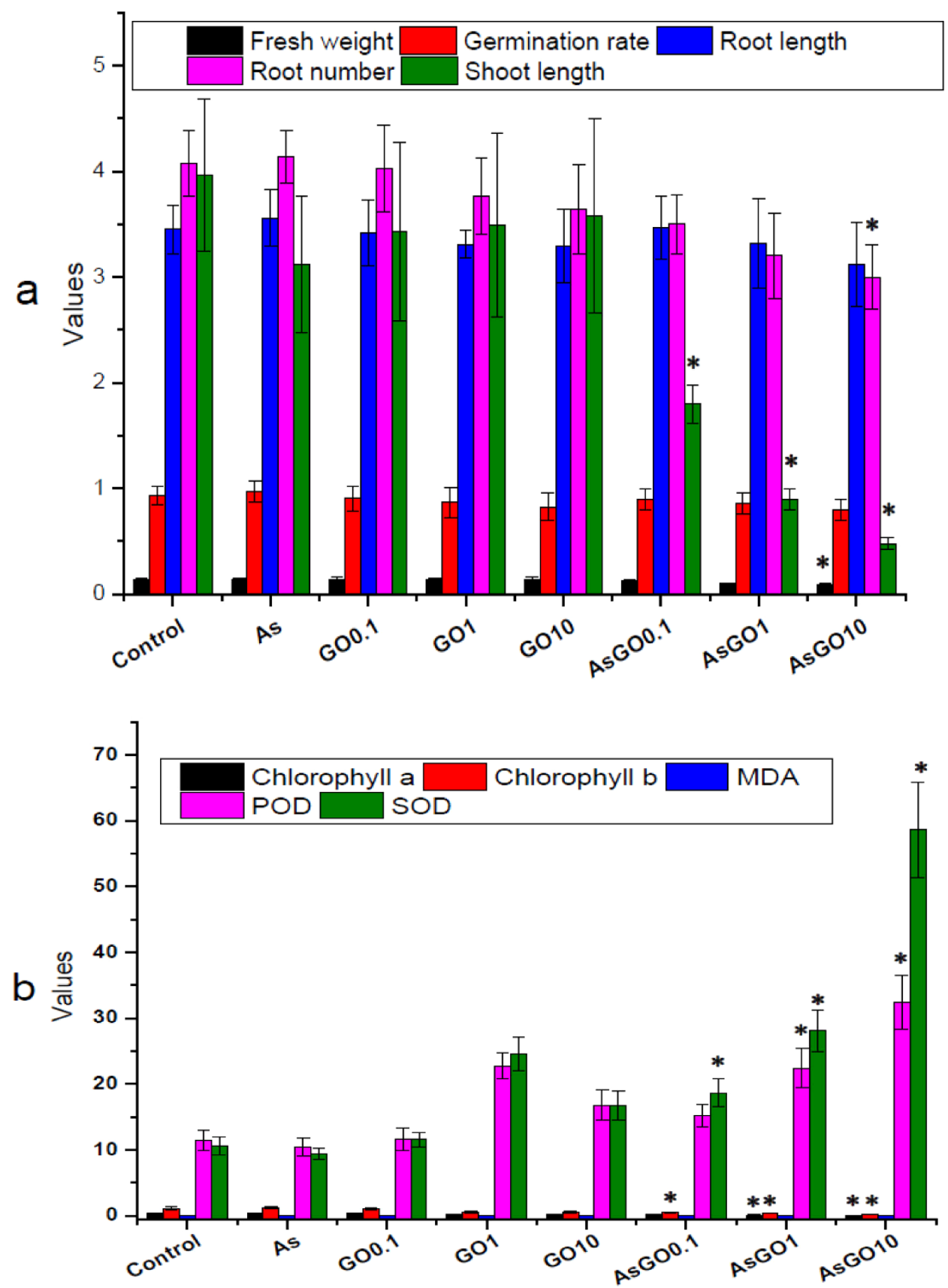

Figure $1 \mid$ Growth inhibition and oxidative effects in wheat exposed to As and GO. Number of replicates, $n=3$. In Figure 1a and b, * represents significant differences $(P<0.05)$ compared with the control and As groups. The units for the enzyme activities of POD and SOD are U/mg/protein and $\mathrm{U} / \mathrm{mg} /$ protein, respectively. The units for chlorophyll a, chlorophyll $\mathrm{b}$ and MDA are mg/g. GO0.1-10, graphene oxide 0.1-10 mg/L; As, arsenic(V), $10 \mathrm{mg} / \mathrm{L}$; POD, peroxidase; SOD, superoxide dismutase; MDA, malondialdehyde.

VIP of more than 1.3. The remaining 48 metabolites made negative contributions to the anabolism of chlorophyll b. AsGO enhanced the contents of the 48 metabolites, and the metabolites with a VIP of more than 1.3 included monopalmitin, galactofuranoside, pentaric acid, propanoic acid, alkane, lysine, serine, threonine, phenylalanine, leucine and isoleucine. The identified high-VIP metabolites are organized in Figure 3, which indicates the effects of AsGO on the main metabolic pathways of cells. Compared with the control and the As-only and GO-only treatments, AsGO inhibited carbohydrate metabolism, enhanced amino acid and secondary metabolism and disturbed fatty acid metabolism and the urea cycle. These results are the first to reveal the mechanisms underlying indirect nanotoxicity using metabolomics.
Promoted damage of the cell structure by uptake of GO and As. To investigate the structural damage to the cell wall and plasma membrane, the Fourier transform infrared spectroscopy (FTIR) spectra and electrolyte leakage in root cells were studied. Compared with the control/As-only/GO-only treatments, AsGO increased the abundance of amino groups, hydroxyl groups, cellulose and polysaccharides on the cell surface (Figure 4a), indicating structural damage to the cell wall and plasma membrane. Electrolyte leakage was further used to detect membrane permeability. As shown in Figure 4b, As and GO did not induce obvious electrolyte leakage. However, AsGO led to significant electrolyte leakage, which increased with the GO concentration. Furthermore, transmission electron microscopy (TEM) images confirmed the structural damage to the cell wall 

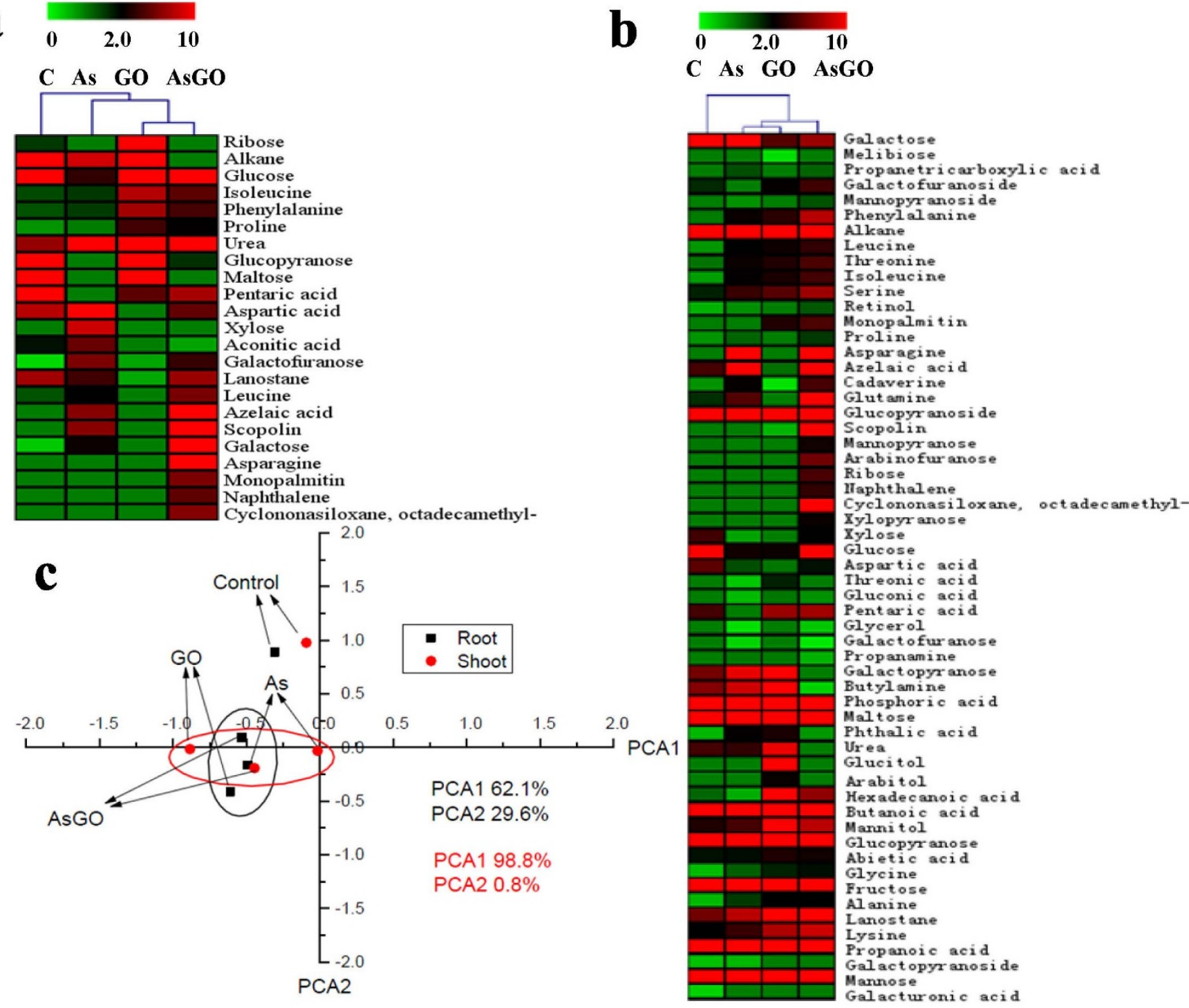

Figure 2 Metabolic analysis of plant cells exposed to GO and As(V). The relative contents of metabolites are represented by heat maps for root (a) and shoot (b) samples. These metabolites show significant differences $(P<0.05)$ between the tested groups. Cluster analysis of the metabolites was carried out using HCL. (c) Cluster analysis of the metabolites using PCA. GO, graphene oxide; HCL, hierarchical clustering; PCA, principal component analysis.

and plasma membrane (Figure 5). The plasma membrane was close to the cell wall in the control, and slight plasmolysis occurred in the As sample. However, remarkable plasmolysis was observed in the GO sample and especially for the AsGO sample, as indicated by the pink arrows in Figure 5a-d. The elliptical outlines of root cells changed to irregular outlines in the GO and AsGO images. In the shoot TEM images from the control, As and GO groups, elliptical chloroplasts adhered to the plasma membrane and regular thylakoids in the chloroplasts were visible. However, the elliptical chloroplasts became circular and were distant from the plasma membrane, and the structure of the thylakoid was damaged in shoot TEM images from the AsGO group, as indicated by the yellow and black arrows in Figure 5e-g. In addition, plasma membrane folding was observed, as indicated with a blue circle in an AsGO image. It is possible that GO increased the permeability of plant cells and then enhanced the uptake of GO and As via passive transport across the plasma membrane. Compared with the control and As samples, remarkable GO deposition was observed in roots exposed to GO and AsGO, as indicated by the green arrows. Compared with GO (Figure 5g), AsGO induced a great deal of GO deposition, which is indicated in the cytoplasm with blue arrows (Figure 5h) for shoot samples.

There is no question of the intracellular uptake of As, but the uptake of GO in plant cells remains unclear. To characterize the uptake of GO, the compositions of the dark dots in plant cells shown in Figure 5d (root) and h (shoot) were characterized using the Raman spectra, as shown in Figure 6. The typical D and G bands of GO located at 1,349 and $1,590 \mathrm{~cm}^{-1}$ were detected in the dark dot compositions of the root samples. In contrast, there were no obvious GO peaks outside of the dark dots. Similar results were found in the shoot samples, although the signals were very weak. These results demonstrated that GO entered the plant cells. Furthermore, TEM-EDX was used to semi-quantitatively analyze the uptake of As (Figure 7). In the root samples, the content of As inside and outside of the dark dots was $0.5 \%$ and $0.1 \%$, respectively. For the shoot samples, the content of As inside and outside of the dark dots was $0.4 \%$ and $0.0 \%$, respectively. The above results demonstrated that As loaded onto GO was taken up via co-transport. In summary, cell structure damage enhanced the uptake of GO and As. 


\section{Carbohydrate

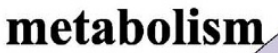 \\ Secondary metabolism}

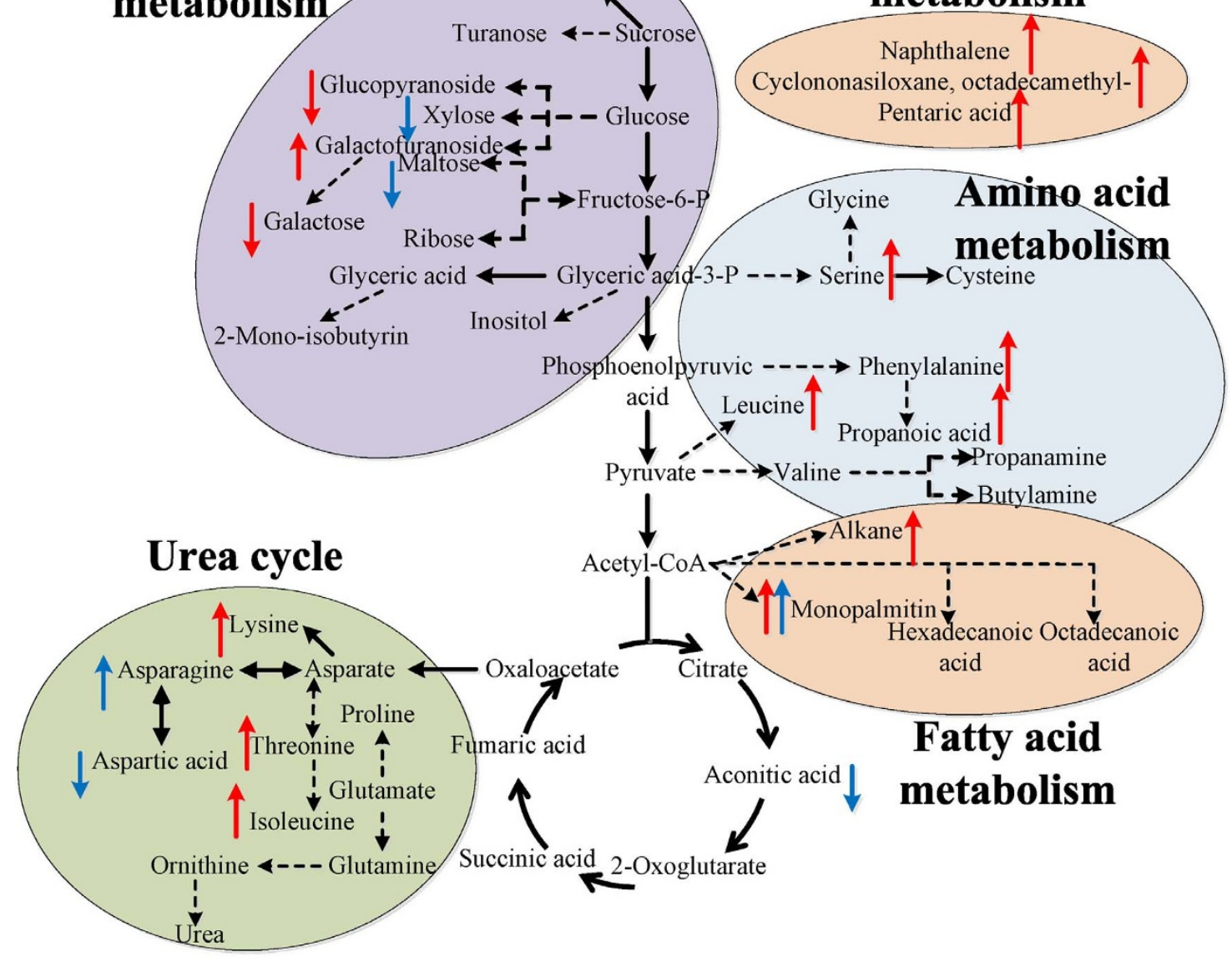

Figure 3 Effects of AsGO on the main metabolic pathways of plant cells. The metabolites labeled with arrows showed significant differences $(P<0.05)$ between the tested groups. Compared with the control, As-only and GO-only groups, AsGO induced metabolic changes with a high VIP in roots and shoots, which are indicated with blue and red arrows, respectively. The directions of the arrows indicate the up-regulation and down-regulation of metabolites. The filled and dotted black arrows represent direct and indirect reactions, respectively. VIP, variable importance for the projection; GO, graphene oxide; As, arsenic.

GO regulates As uptake and transformation through genes and enzymes. The uptake and transformation of $\mathrm{As}(\mathrm{V})$ were further quantified using the liquid chromatograph-inductively coupled plasma mass spectrometer (HPLC-ICP-MS). The uptake rate of As was high in the plants, as shown in Figure 8a. Compared with the $19.9 \%$ rate of As uptake recorded without GO exposure, the uptake rate of As increased to $24.6 \%$ and $32.1 \%$, then decreased to $16.1 \%$ in the presence of GO concentrations of $0.1,1$ and $10 \mathrm{mg} / \mathrm{L}$, respectively. Therefore, the regulation of uptake of As by GO was concentration dependent. Both of $\mathrm{As}(\mathrm{V})$ and $\mathrm{As}(\mathrm{III})$ were detected in all groups, suggesting transformation of $\mathrm{As}(\mathrm{V})$ to $\mathrm{As}(\mathrm{III})$ in vivo. Moreover, DMA was detected in the group exposed to $\mathrm{As}(\mathrm{V})$ alone. GO enhanced the accumulation ratio of As(III) in the roots, with root-to-shoot concentration ratio of 14.0, 10.9, 16.8 and 22.1 being observed in the As, AsGO 0.1, AsGO 1 and AsGO 10 groups, respectively. The accumulation ratio for $\mathrm{As}(\mathrm{V})$ was 4.2, 9.1, 11.4 and 7.2 in As, AsGO 0.1, AsGO 1 and AsGO 10 groups, respectively, showing concentration dependence. In summary, the generation of DMA to detoxify inorganic As was inhibited by GO, and GO led to the accumulation of high-toxicity As(III) in wheat roots.

To identify roles of direct chemical regulation in As uptake and transformation by GO, the interaction of GO with $\mathrm{As}(\mathrm{V})$ in the aqueous phase was investigated (Figure 8b). In the As-only group, $13.4 \%$ of $\mathrm{As}(\mathrm{V})$ was transformed to $\mathrm{As}(\mathrm{III})$. In the other groups, GO did not significantly alter transformation of As(V). However, 73.5-
$76.6 \%$ of the transformed As(III) was absorbed on GO, and the rate of transformation was not significantly different in the different groups. The ratio of absorbed As to total As increased with the GO concentration, with values of $3.1,8.9$ and $12.1 \%$ being obtained in GO 0.1, GO 1 and GO 10, respectively. These results showed that As, especially As(III), was adsorbed onto GO, but GO played a limited role in transformation of As through direct chemical reactions. To further identify the functions of biological regulation in As uptake and transformation by GO, the intracellular activities of key enzymes and gene expression were quantified, as shown in Figure 8c. GO at low and moderate concentrations $(0.1$ and $1 \mathrm{mg} / \mathrm{L})$ enhanced the expression of phosphate transporter genes that transport As through the plasma membrane, but their expression decreased under a high concentration of $10 \mathrm{mg} / \mathrm{L}$ GO. The activity of arsenate reductase, which plays an important role in transformation of $\mathrm{As}(\mathrm{V})$ to As(III), showed similar changes to the expression of phosphate transporter genes. The activity of arsenate reductase was enhanced and then inhibited by increasing GO concentrations. These results demonstrated that $\mathrm{GO}$ regulated As uptake and transformation through both chemical and biological processes and this regulation was concentration dependent.

\section{Discussion}

In general, GO is thought to be relatively biocompatible compared with metal nanoparticles and other carbon nanomaterials ${ }^{3,30-32}$. No 

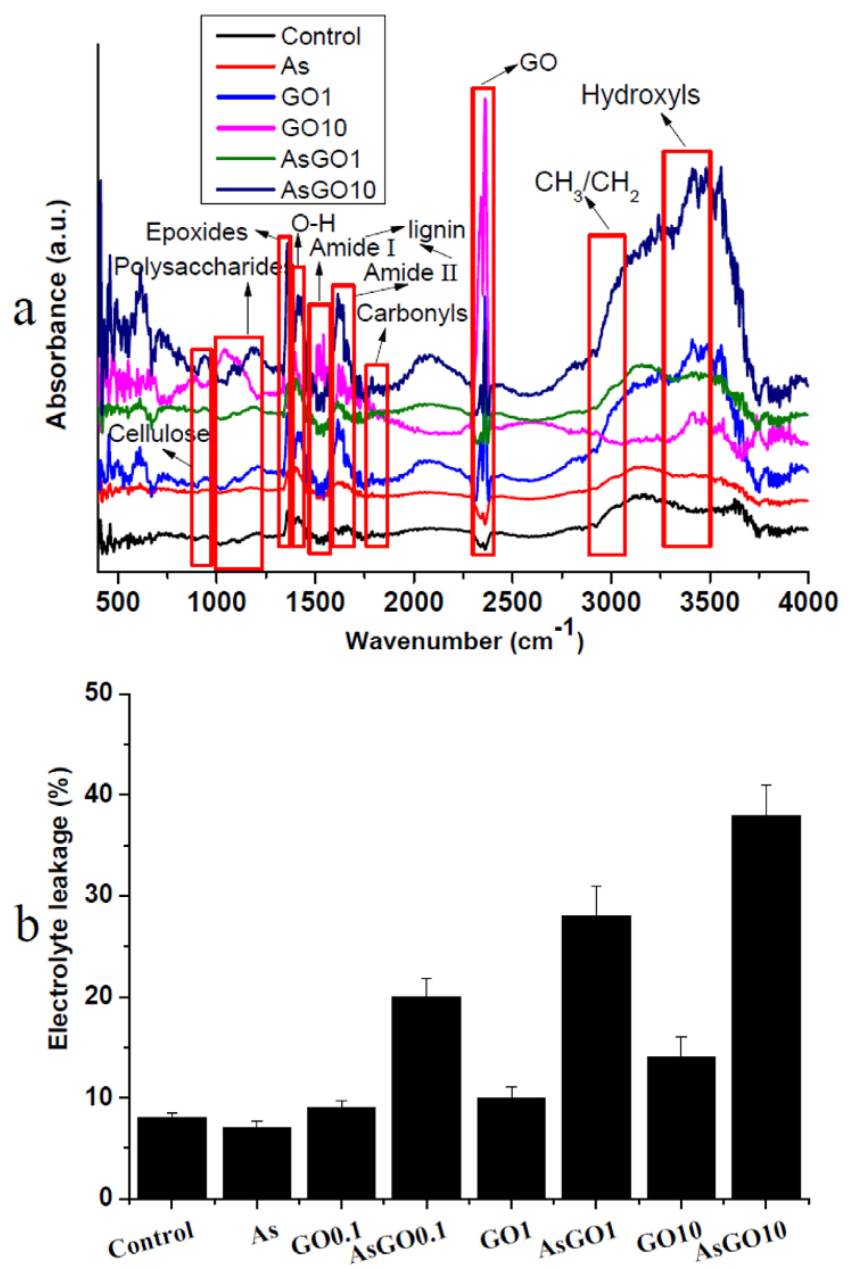

Figure $4 \mid$ Structural damage to the cell wall and plasma membrane. Fourier transform infrared spectra (a) and electrolyte leakage (b) in root cells. GO10 and GO1 are cells exposed to 10 and $1 \mathrm{mg} / \mathrm{L} \mathrm{GO}$, respectively. As, arsenic (V) $10 \mathrm{mg} / \mathrm{L}$; GO, graphene oxide. The chemical groups in the Fourier transform infrared spectra refer to previous work ${ }^{25-27}$.

adverse effects of GO on the growth and biomass of higher plants, such as cabbage, tomato, red spinach and lettuce, have been observed at concentrations below $400 \mathrm{mg} / \mathrm{L}^{5,10}$. Treatment with $\mathrm{GO}$ dosages ranging from 0.1 to $10 \mathrm{mg} / \mathrm{L}$ does not significantly disturb seed germination in wheat, as demonstrated based on the growth, biomass and oxidative stress of the plants. Although GO slightly reduced root growth, the reduction was not remarkable. The amino groups on biological cells could interact with GO, resulting in GO wrapping or adsorbing around cells; the cells were then isolated from the culture medium, leading to a reduction in plant growth ${ }^{33}$. Treatment with $\mathrm{As}(\mathrm{V})$ at a concentration of $10 \mathrm{mg} / \mathrm{L}$ also did not induce significant adverse effects, but considerable adverse effects were observed when wheat was exposed to $\mathrm{As}(\mathrm{V})$ and GO together, even at a very low dose $(0.1 \mathrm{mg} / \mathrm{L})$ of $\mathrm{GO}$. Compared with that in the control, the application of $0.1 \mathrm{mg} / \mathrm{L}$ of GO with As triggered a reduction of shoot length and chlorophyll b contents and a increase of SOD activity. Similarly, when the 24-h mortality of Ceriodaphnia dubia was assessed, 2.4-3.2 mg/L As(V) led to an increase from $2.5-37.5 \%$ without nanoparticles to $43-65 \%$ in the presence of $10-50 \mathrm{mg} / \mathrm{L}$ nano- $\mathrm{Al}_{2} \mathrm{O}_{3}$ and nano- $\mathrm{Al}_{2} \mathrm{O}_{3}{ }^{34}$. Given that As contamination is widespread in agricultural plants such as wheat, rice and $\operatorname{corn}^{21,22}$, the impact of "indirect" nanotoxicity on food safety should be considered.

The potential benefits of applying metabolomics to reveal molecular mechanisms of nanotoxicology and nanomedicine are only start-

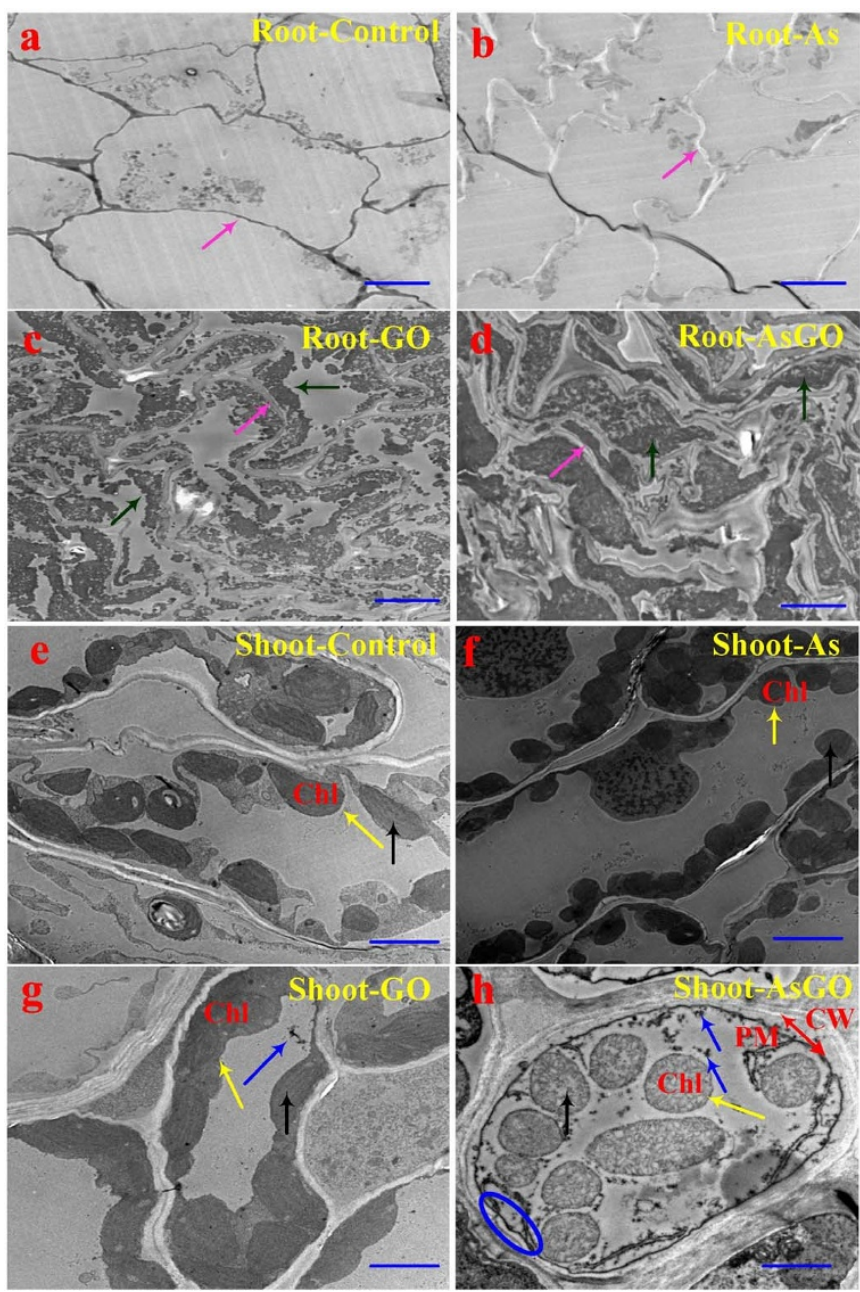

Figure 5 | Transmission electron microscopy images of plant cells. Pink arrows indicate the cell wall in (a)-(d). Green arrows indicate GO deposition in the cytoplasm for (c) and (d). Yellow arrows indicate chloroplasts in (e)-(h). Black arrows indicate thylakoids in (e)-(h). Blue arrows indicate GO deposition in the cytoplasm for $(\mathrm{g})$ and $(\mathrm{h})$ images. The blue circle indicates the plasma membrane in (h). Cw, cell wall; $\mathrm{Pm}$, plasma membrane; Chl, chloroplast; Thy, thylakoid. Scale bar, $5 \mu \mathrm{m}$.

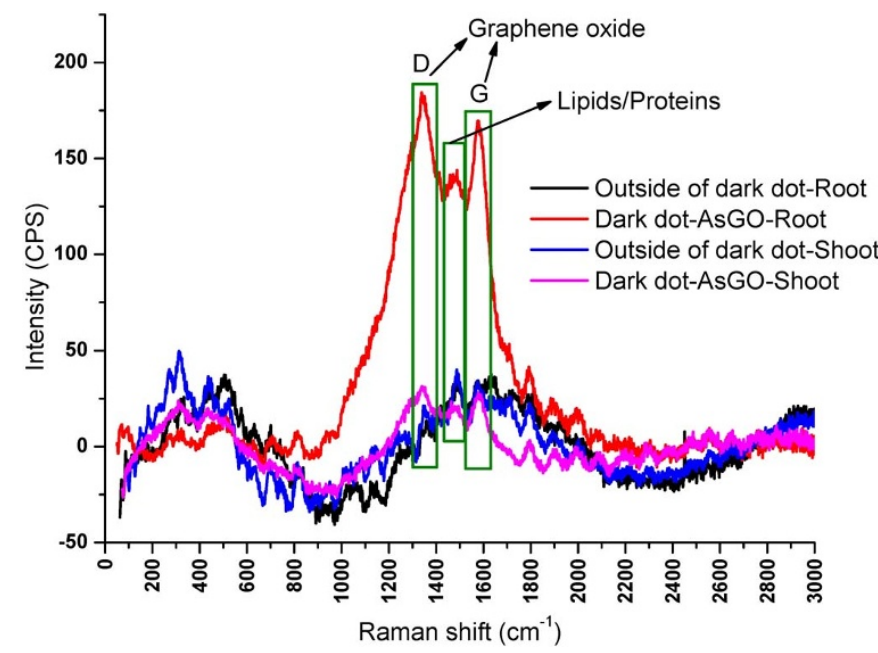

Figure 6 | Raman spectra of the dark dot compositions of the plant cells shown in Figure 5d (root) and h (shoot) exposed to AsGO. GO, graphene oxide. The chemical groups in the Raman spectra refer to previous work $^{28,29}$. 

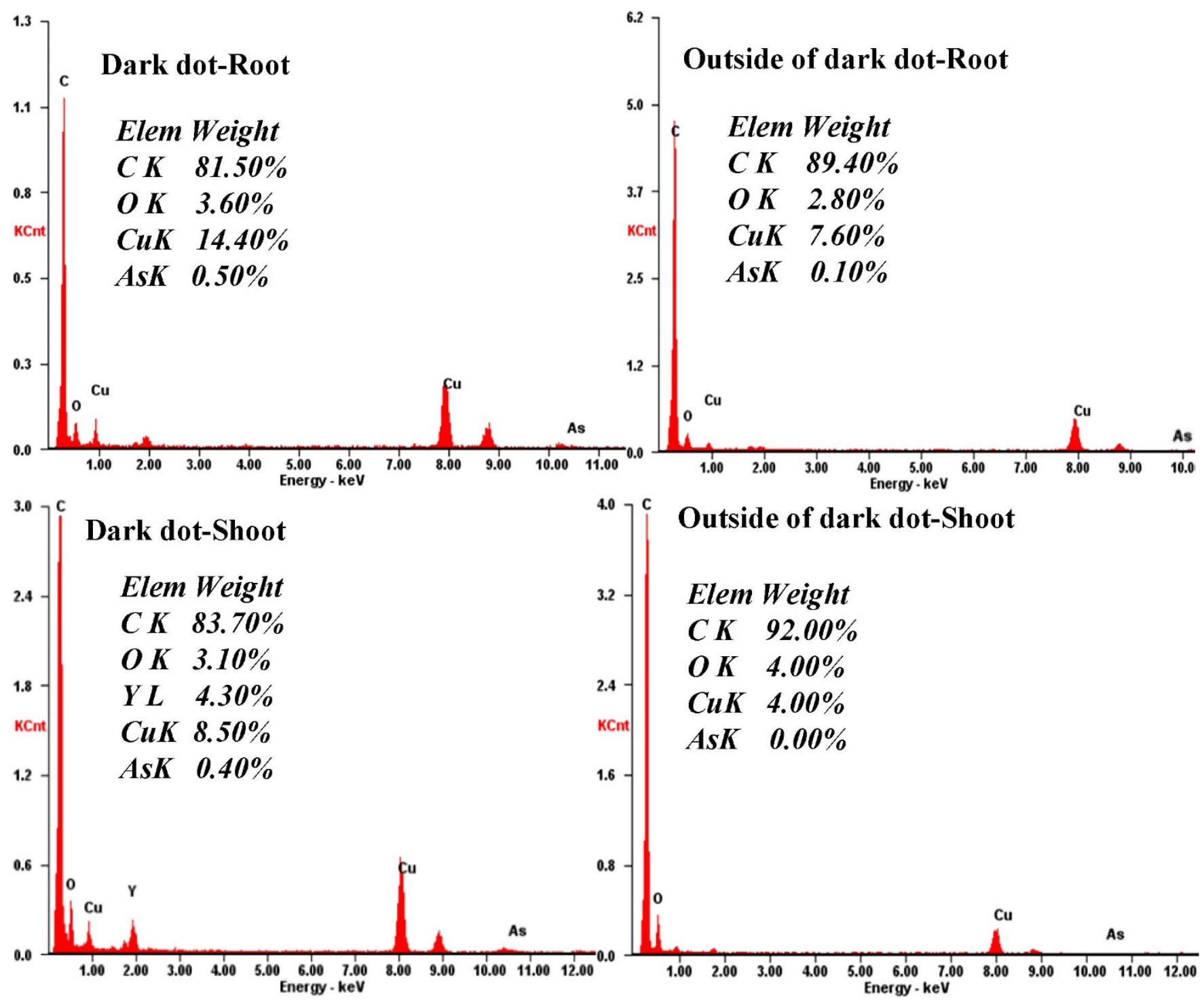

Figure 7 | Energy dispersive spectra of the dark dot compositions of the plant cells from Figure 5d (root) and h (shoot) exposed to AsGO.

ing to be realised ${ }^{3,35}$. Compared with the control/As-only/GO-only treatments, AsGO inhibited carbohydrate metabolism, except for galactofuranoside. The inhibition of carbohydrate metabolism was linked to the insufficiency of energy supplementation, which was consistent with growth inhibition. Carbohydrate metabolism is also considered to be an indicator of osmoprotection ${ }^{36}$. Thus, the results depicted in Figure $4 \mathrm{~b}$ indicate that AsGO increased cellular electrolyte leakage. There is extensive and unequivocal evidence that amino acid metabolism and secondary metabolism in plants are associated with the cellular response to the stress ${ }^{37-39}$. AsGO enhanced amino acid and secondary metabolism, including that of leucine, glycine, phenylalanine, naphthalene and octadecamethyl-cyclononasiloxane. Compared with that in the other groups, AsGO significantly enhanced the accumulation of a saturated fatty acid (alkane). Increases in saturated fatty acids are linked to the reduction of membrane fluidity ${ }^{40}$, which most likely led to the membrane structure damage depicted in Figures 4 and 5. AsGO also disturbed the urea cycle and induced the accumulation of amino acids, such as lysine, threonine, asparagine and isoleucine. Specifically, the pathway of nitrogen storage was altered. These metabolic responses may be helpful for understanding the molecular mechanisms underlying toxicity amplification from the co-exposure of $\mathrm{As}(\mathrm{V})$ and $\mathrm{GO}$ to plants.
The mechanisms of the uptake of GO, and even carbon nanomaterials, into plant cells remain obscure at present. Fullerenes up to $100 \mathrm{~nm}$ in diameter enter the gaps between the cell wall and the plasma membrane $e^{41}$, suggesting that nanomaterials can penetrate a cell wall even when they are larger than the cell wall pores ${ }^{41}$. As shown in Figure $5 \mathrm{c}$ and d, uptake of GO was observed in TEM images and was confirmed by the Raman spectra presented in Figure 6. Generally, the uptake of nanomaterials is increased by the enhancement of permeability ${ }^{41}$. AsGO triggered structural damage to the cell wall and plasma membrane and increased electrolyte leakage (Figure 4), which generally occurred for very high concentrations (more than $100 \mathrm{mg} / \mathrm{L}$ ) of GO exposure to cells ${ }^{31,32}$. Moreover, Figure $5 \mathrm{c}$ and d demonstrates that plasmolysis occurred. It has been proposed that carbon nanotube and nano- $\mathrm{ZnO}$ materials enter the roots of seedlings by increasing the permeability of plant cell walls ${ }^{42}$. After the uptake of GO and As, the damage and structural alterations observed in organelles were considerable, including destruction of chloroplasts, which corresponded to a decrease of chlorophyll biosynthesis.

Another important finding was that As accumulated on GO in cells (Figure 7), demonstrating that GO increased the uptake of As via co-transport. Figure 8 a shows that 0.1 and $1 \mathrm{mg} / \mathrm{L} \mathrm{GO}$ enhanced the uptake of As, whereas $10 \mathrm{mg} / \mathrm{L}$ GO reduced As uptake. 

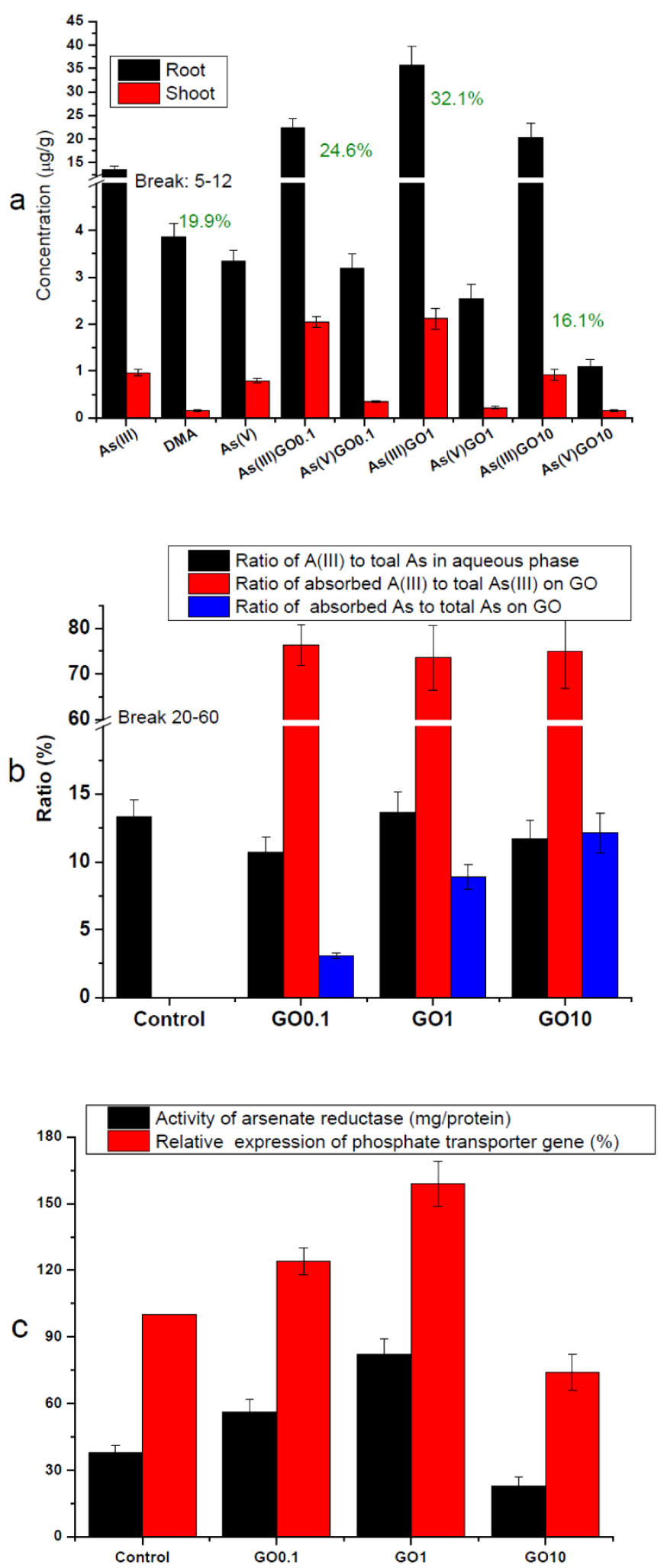

Figure 8 | GO-regulated As uptake and transformation. GO-regulated As uptake and transformation in roots and shoots (a). GO-regulated As uptake and transformation in the aqueous phase (b). GO-regulated activity of arsenate reductase and the expression of phosphate transporter genes (c). GO, graphene oxide; AsGO0.1-10, 10 mg/L As(V) with GO ranged from $0.1-10 \mathrm{mg} / \mathrm{L}$. DMA, dimethylarsinate.
Concerning direct chemical interactions, our results showed that As, and especially As(III), could be absorbed on GO, and the absorption increased with the GO concentration (Figure $8 \mathrm{~b}$ ). These findings implied that the uptake of As involved pathways other than chemical interactions alone. It has been reported that As is taken up by plants via phosphate transporters ${ }^{43}$. Recently, the genotoxicity of graphenebased materials has attracted much attention ${ }^{32,41}$. Figure $8 \mathrm{c}$ shows that low concentrations of GO increased the expression of phosphate transporter genes, while high concentrations of GO decreased their expression. Therefore, GO influenced the uptake of As via at least three pathways: permeability regulation, co-transport and transporter gene regulation. DMA is a less toxic methylated form of inorganic $\mathrm{As}^{44}$. GO inhibited the formation of DMA in plants and then enhanced the phytotoxicity of As, as shown in Figures 1 and $8 \mathrm{a}$. As(III) is more toxic in terms of ecological and health risks than $\mathrm{As}(\mathrm{V})^{45}$. Figure 8a shows that the ratio of As(III) to As(V) first increased and then decreased with the GO concentration in both the roots and shoots. Concerning direct chemical reactions, as indicated in Figure $8 \mathrm{~b}, \mathrm{GO}$ increased the transformation of $\mathrm{As}(\mathrm{V})$ to As(III), but there was no significant change associated with the GO concentration. Arsenate reductase activity is considered to play a critical role in the transformation of $\mathrm{As}(\mathrm{V})$ to $\mathrm{As}(\mathrm{III})$ in plants ${ }^{44,46}$. GO regulated the transformation of As through arsenate reductase activity (Figure 8c). Therefore, GO regulated the uptake and transformation of As through chemical and biological processes together.

In conclusion, "indirect" nanotoxicity is defined as toxic amplification of other toxicants or pollutants by nanomaterials. This work comprehensively analyzed "indirect" nanotoxicity of the relatively biocompatible GO, which amplified the phytotoxicity of widespread As in wheat. The indirect nanotoxicity of GO involved at least five components, as described in Figure S7: (i) enhancement of growth inhibition and oxidative stress; (ii) regulation of key forms of metabolism, such as carbohydrate, amino acid and secondary metabolism; (iii) increasing damage to cell structures and permeability; (iv) regulation of As uptake and transformation via chemical interactions, such as through As loading onto GO entering the cell; and (v) regulation of As uptake and transformation via biological interactions, such as transporter gene expression and enzyme catalysis. Given the widespread exposure to As in agriculture, "indirect" nanotoxicity of GO should be considered in relation to food safety.

\section{Methods}

Characteristics of GO nanosheets. GO nanosheets were obtained from Nanjing XFNANO Materials Tech Co., Ltd., China. XPS measurements were conducted using the Axis Ultra XPS system (Axis Ultra DLD, Kratos) with a monochromatic Al K $\alpha$ Xray source $(1486.6 \mathrm{eV})$. The spectra were analyzed with Casa-XPS V2.3.13 software. Samples were prepared and dispersed in absolute ethanol, and atomic force microscopy (AFM) and field emission TEM measurements were then conducted on a Veeco Nanoscope 4 or JEM-2010 FEF, respectively. UV spectra were obtained using a TU-1901 spectrophotometer with UVWin5 software. The size distribution was detected through wide-angle light scattering (BI-200SM, Brookhaven). Raman spectrometry (Thermo Scientific, DXR) with a $514 \mathrm{~nm}$ laser was used to analyze GO structure.

Seed germination experiment. Wheat (Triticum aestivum L.) seeds were sterilized for 20 min using $2 \% \mathrm{H}_{2} \mathrm{O}_{2}$. Subsequently, the seeds were completely rinsed using pure water $(18.2 \Omega / \mathrm{cm}$ ). Double-layer filter paper (diameter, $9 \mathrm{~cm}$ ) was placed in a culture dish. In a same volume of $6 \mathrm{~mL}, 10 \mathrm{mg} / \mathrm{L} \mathrm{As}(\mathrm{V}) ; 0.1-10 \mathrm{mg} / \mathrm{L} \mathrm{GO} ; 10 \mathrm{mg} / \mathrm{L} \mathrm{As}(\mathrm{V})$ plus $0.1-10 \mathrm{mg} / \mathrm{L} \mathrm{GO}$; and pure water were added to different culture dishes, respectively. In each culture dish, 15 seeds with the same diameter and shape were germinated. For uniformity, the seeds were arranged on the plates with consistent growth spacing. The culture dishes were placed in a climate chamber at $3000 \mathrm{Lx}$ irradiation under a temperature of $24^{\circ} \mathrm{C}$ temperature $80 \%$ humidity.

Plant growth and oxidative stress. To avoid the influence of other factors, the culture medium, temperature and spacing were kept uniform for all groups, such that these factors did not have adverse effects on plant growth during germination. To reduce differences among manipulations, four dishes were prepared for each group. Germination was performed for eight days. The fresh weight, leaf length, root length and root number were recorded using a plant analyzer (EPSON PerfectionV700 Photo, SilverFast STD4800). The content of chlorophyll a, the activities of SOD/POD and content of MDA were analyzed using a TU-1901 spectrophotometer, as described 
previously ${ }^{47}$. The concentration of lipid peroxides was quantified in terms of the MDA concentration. As monitored at $560 \mathrm{~nm}$, SOD activity was assayed by measuring the ability of the enzyme extract to inhibit the photochemical reduction of nitrotetrazolium blue chloride. After the enzyme extract reacted with $\mathrm{H}_{2} \mathrm{O}_{2}$, POD activity was measured based on the $\mathrm{H}_{2} \mathrm{O}_{2}$ decomposition rate, using guaiacol as a hydrogen donor, and CAT activity was estimated based on the decrease in absorbance at $240 \mathrm{~nm}$.

Metabolic analyses. To obtain representative material from large plant tissues, root and shoot tips (length, $1 \mathrm{~cm}$ ) were collected from more than 30 random plants using a sharp knife. To avoid metabolic alterations during preparation, the root and shoot tips $(0.1 \mathrm{~g})$ were snap-frozen in liquid nitrogen and then ground with $2 \mathrm{~mL}$ of a solution of methanol: chloroform: water (volumetric ratio $=2.5: 1: 1$ ) and $0.2 \mathrm{mg} /$ $\mathrm{mL}$ ribitol $(50 \mu \mathrm{L})$ as an internal standard. Metabolites were intensively extracted using ultrasound ( $200 \mathrm{~W}, 30 \mathrm{~min}$ ) in an ice bath, then centrifuged for $10 \mathrm{~min}$ at $9,000 \mathrm{~g}$ at $4^{\circ} \mathrm{C}$. The supernatant was subsequently collected, and the sediment was extracted again using $1 \mathrm{~mL}$ of a methanol:chloroform solution (volumetric ratio = $1: 1$ ), after which ultrasound and centrifugation were performed as described above. This supernatant was mixed with the previously collected supernatant, and water $(500 \mu \mathrm{L}$ ) was added, followed by centrifugation at $9,000 \mathrm{~g}$ for $5 \mathrm{~min}$. The lower phase was dried through nitrogen blow-off. For the upper phase, methanol was removed via nitrogen blow-off, and the remaining water was lyophilized. Methoxamine hydrochloride $(20 \mathrm{mg} / \mathrm{mL}, 50 \mu \mathrm{L})$ and $\mathrm{N}$-methyl-N-

(trimethylsilyl)trifluoroacetamide $(80 \mu \mathrm{L})$ were added as derivatives. Samples $(1 \mu \mathrm{L})$ were injected into the gas chromatography column in split mode $(1: 25)$. Gas chromatography (Agilent 6890N, Agilent Technologies, USA) linked with quadruple mass spectrometry (Agilent 5973, Agilent Technologies, USA) was applied to analyze metabolites. The detection and identification of metabolites were performed in a previous study by our group ${ }^{48}$.

Electron microscopy and spectroscopy analysis. TEM was used to investigate the cellular ultrastructure. Tips of roots and shoots were thoroughly washed using water, prefixed in $2.5 \%$ glutaraldehyde, postfixed in $1 \%$ osmium tetroxide for $2 \mathrm{~h}$, dehydrated in a graded ethanol series and then embedded in epoxy resin (ETON 812). Ultrathin sections $(80 \mathrm{~nm})$ were cut using an ultramicrotome with a diamond knife (Leica EM FC7). TEM images were obtained on Hitachi HT7700 TEM. TEM coupled EDX (JEM-2010FEF, phoenix $60 \mathrm{t}$ ) was conducted to detect elements in the roots and shoots. The operation parameters were as follows: $200.00 \mathrm{kV}, 45.00$ azimuth, 51.2 ampT Sutw-sapphire detector type, with a resolution of 137.25. A Raman spectrometer was used to analyze the GO compositions in the cells, which were recorded on a Thermo Scientific DXR Raman microscope using $780 \mathrm{~nm}$ excitation from a diode-pumped, solid state (DPSS) laser. FTIR was conducted to analyze the chemical groups on the cellar surface, and the results were recorded on a Bruker Tensor 27 infrared spectrometer with a resolution of $2 \mathrm{~cm}^{-1}$ at $4,000-400 \mathrm{~cm}^{-1}$. A measured amount of biomass was mixed with potassium bromide (mass ratios $=$ $2: 98$ ). The mixture was ground into fine particles and compressed into a translucent sample disk with a manual hydraulic press. The disks were then fixed in the FTIR apparatus for analysis.

Electrolyte leakage. Electrolyte leakage was measured to detect membrane permeability and assessed as described in a previous report ${ }^{49}$. Root samples were washed three times using pure water to remove surface-adhered electrolytes. The roots were placed in closed vials containing $10 \mathrm{~mL}$ of pure water and incubated at $25^{\circ} \mathrm{C}$ on a shaker at $500 \mathrm{rmp}$ for $24 \mathrm{~h}$, after which the electrical conductivity of the solution $\left(\mathrm{EC}_{0}\right)$ was determined. The samples were then autoclaved at $120^{\circ} \mathrm{C}$ for $20 \mathrm{~min}$, and the final electrical conductivity $\left(\mathrm{EC}_{\mathrm{t}}\right)$ was obtained after equilibration at $25^{\circ} \mathrm{C}$. Electrolyte leakage was defined as follows: electrolyte leakage $(\%)=\left[\mathrm{EC}_{0} / \mathrm{EC}_{\mathrm{t}}\right]$ $\times 100$.

Detection of As uptake and transformation. Samples of the roots and shoots with a fresh weight of $0.1 \mathrm{~g}$ were lyophilized. The samples were then ground in $5 \mathrm{~mL}$ of methanol and water (volume ratio, $2: 1$ ). As was extracted using ultrasound at $150 \mathrm{~W}$ for $30 \mathrm{~min}$. Subsequently, the samples were centrifuged at $8,000 \mathrm{~g}$ for $15 \mathrm{~min}$, and the supernatant was collected. The sediments were extracted and centrifuged again, and the two supernatants were combined. After filtration through a $0.22 \mu \mathrm{m}$ membrane, $1 \mu \mathrm{L}$ of the sample was injected into HPLC-ICPMS (Agilent 1260/7700X). As a sub-experiment, the direct interactions of GO with As were studied in the aqueous phase. $\mathrm{As}(\mathrm{V})$ at $10 \mathrm{mg} / \mathrm{L}$ reacted with a $\mathrm{GO}$ at $0.1-$ $10 \mathrm{mg} / \mathrm{L}$ in the same incubator as was used for seed germination. The suspension was filtered to separate GO (diameter, $465-486 \mathrm{~nm}$, center at $476 \mathrm{~nm}$ ) with an ultrafilter (3KD, approximately $10 \mathrm{~nm}$ pore, Millipore). The filtrate was directed analyzed using HPLC-ICP-MS. The adsorbed As was successively eluted using $0.5 \mathrm{~mL}$ of $1 \mathrm{M} \mathrm{NaOH}, 0.5 \mathrm{~mL}$ of $1 \mathrm{M} \mathrm{HCl}$ and $0.5 \mathrm{~mL}$ of water. The eluent was analyzed through HPLC-ICP-MS. In all samples, $\mathrm{As}(\mathrm{V}), \mathrm{As}(\mathrm{III})$, dimethylarsinate and monomethylarsinate were detected.

Arsenate reductase activity. Arsenate reductase activity was assayed using the coupled enzymatic reaction described by Duan et $\mathrm{a}^{46}$. The assay was performed in $50 \mathrm{mM} 3$-(N-morpholino) propanesulfonic acid, $50 \mathrm{mM} \mathrm{2-( \textrm {N } -}$

morpholino)ethanesulfonic acid, $\mathrm{pH} 6.5$, containing $1.5 \mathrm{mM}$ nicotinamide adenine dinucleotide phosphate, 1 unit of yeast glutaredoxins, $1 \mathrm{mM}$ glutathione and $10 \mathrm{mM}$ sodium arsenate, in a total volume of $1.5 \mathrm{~mL}$. All of the measurements were performed at $30^{\circ} \mathrm{C}$. The oxidation of nicotinamide adenine dinucleotide phosphate was monitored by recording the decrease in absorbance using a TU-1901 spectrophotometer with UVWin5 software.

Expression of phosphate transporter genes. The analysis of phosphate transporter gene expression was described in detail previously ${ }^{50}$. Briefly, based on known highaffinity phosphate transporter sequences, degenerate polymerase chain reaction (PCR) primers were designed against highly conserved regions identified through sequence alignment. A combination of two upstream primers (5'-TTYTTYCANGAYGCNTAYGAY- $3^{\prime}$ and $5^{\prime}$-GTNCCNGGNTAYTGGTTYCANGT-3') and a downstream primer (5'-GGNCCRAARTTNGCRAARAA-3') were chosen for PCR. For phosphate transporter sequences, first-strand cDNA synthesis was performed on $1 \mu \mathrm{g}$ of total RNA as a template with 5 pmol of the antisense primer $5^{\prime}$ -

GGNCCRAARTTNGCRAARAA- $3^{\prime}$. For 18S RNA reactions, the antisense primer 5'-CACTTCACCGGACCATTCAATCG-3' was employed for first-strand synthesis using $0.5 \mu \mathrm{g}$ of total RNA as a template.

Statistical analyses. All treatments included three replicates, and the mean \pm SD (standard deviation) is presented with error bars. A t-test with Welch approximation for unequal group variances with $P$-values based on the t-distribution was performed with a cutoff of $P<0.05$ in Multiple Experiment Viewer 4. PCA and OPLS-DA were performed using SIMCA-P 11.5 software. A heat map was generated using MeV 4.8.1 software. The default distance metric for HCL was Pearson correlation, and the linkage method selection was average linkage clustering.

1. Dreyer, D. R., Park, S., Bielawski, C. W. \& Ruoff, R. S. The chemistry of graphene oxide. Chem. Soc. Rev. 39, 228-40 (2010).

2. Novoselov, K. S. et al. A roadmap for graphene. Nature 490, 192-200 (2012).

3. Hu, X. \& Zhou, Q. Health and ecosystem risks of graphene. Chem. Rev. 113, 3815-35 (2013).

4. Seabra, A. B., Paula, A. J., de Lima, R., Alves, O. L. \& Duran, N. Nanotoxicity of graphene and graphene oxide. Chem. Res. Toxicol. 27, 159-168 (2014).

5. Begurn, P., Ikhtiari, R. \& Fugetsu, B. Graphene phytotoxicity in the seedling stage of cabbage, tomato, red spinach, and lettuce. Carbon 49, 3907-3919 (2011).

6. Nair, R. et al. Effect of carbon nanomaterials on the germination and growth of rice plants. J. Nanosci. Nanotechno. 12, 2212-2220 (2012).

7. Zhao, L. et al. Effect of surface coating and organic matter on the uptake of $\mathrm{CeO}_{2}$ NPs by corn plants grown in soil: Insight into the uptake mechanism. J. Hazard. Mater. 225, 131-138 (2012)

8. Ruiz, O. et al. Graphene oxide: A nonspecific enhancer of cellular growth. ACS Nano 5, 8100-8107 (2011).

9. Yang, K. et al. In vivo biodistribution and toxicology of functionalized nanographene oxide in mice after oral and intraperitoneal administration. Biomaterials 34, 2787-2795 (2013).

10. Anjum, N. A. et al. Single-bilayer graphene oxide sheet impacts and underlying potential mechanism assessment in germinating faba bean (Vicia faba L.). Sci. Total Environ. 472, 834-841 (2014).

11. De La Torre-Roche, R. et al. Multiwalled carbon nanotubes and $\mathrm{C}_{60}$ fullerenes differentially impact the accumulation of weathered pesticides in four Aagricultural plants. Environ. Sci. Technol. 47, 12539-12547 (2013).

12. Baun, A., Sorensen, S. N., Rasmussen, R. F., Hartmann, N. B. \& Koch, C. B. Toxicity and bioaccumulation of xenobiotic organic compounds in the presence of aqueous suspensions of aggregates of nano- $\mathrm{C}_{60}$. Aquat. Toxicol. 86, 379-387 (2008).

13. Schwab, F. et al. Diuron sorbed to carbon nanotubes exhibits enhanced toxicity to Chlorella vulgaris. Environ. Sci. Technol. 47, 7012-7019 (2013).

14. Hu, J. Wang, D. Forthaus, B. E. \& Wang, J. Quantifying the effect of nanoparticles on $\mathrm{As}(\mathrm{V})$ ecotoxicity exemplified by nano- $\mathrm{Fe}_{2} \mathrm{O}_{3}$ (magnetic) and nano- $\mathrm{Al}_{2} \mathrm{O}_{3}$. Environ. Toxicol. Chem. 31, 2870-2876 (2012).

15. Cohen, S. M., Arnold, L. L., Beck, B. D., Lewis, A. S. \& Eldan, M. Evaluation of the carcinogenicity of inorganic arsenic. Crit. Rev. Toxicol. 43, 711-752 (2013).

16. Zhang, J. et al. Graphene oxide as a matrix for enzyme immobilization. Langmuir 26, 6083-6085 (2010)

17. Chandra, V. et al. Water-dispersible magnetite-reduced graphene oxide composites for arsenic removal. ACS Nano 4, 3979-3986 (2010).

18. Hu, X., Mu, L., Wen, J. \& Zhou, Q. Covalently synthesized graphene oxideaptamer nanosheets for efficient visible-light photocatalysis of nucleic acids and proteins of viruses. Carbon 50, 2772-2781 (2012).

19. Miralles, P., Johnson, E., Church, T. L. \& Harris, A. T. Multiwalled carbon nanotubes in alfalfa and wheat: Toxicology and uptake. J. R. Soc. Interface $\mathbf{9}$, 3514-3527 (2012).

20. Rico, C. M., Majumdar, S., Duarte-Gardea, M., Peralta-Videa, J. R. \& GardeaTorresdey, J. L. Interaction of nanoparticles with edible plants and their possible implications in the food chain. J. Agric. Food Chem. 59, 3485-3498 (2011).

21. Cao, Q., Hu, Q. H., Baisch, C., Khan, S. \& Zhu, Y. G. Arsenate toxicity for wheat and lettuce in six Chinese soils with different properties. Environ. Toxicol. Chem. 28, 1946-1950 (2010).

22. Bhattacharya, P., Samal, A. C., Majumdar, J. \& Santra, S. C. Arsenic contamination in rice, wheat, pulses, and vegetables: A study in an arsenic affected area of West Bengal, India. Water Air Soil Pollut. 213, 3-13 (2010). 
23. Ferrari, A. C. et al. Raman spectrum of graphene and graphene layers. Phys. Rev. Lett. 97, 187401 (2006).

24. Akhavan, O. \& Ghaderi, E. Graphene nanomesh promises extremely efficient in vivo photothermal therapy. Small 9, 3593-3601 (2013).

25. Kudin, K. N. et al. Raman spectra of graphite oxide and functionalized graphene sheets. Nano Lett. 8, 36-41 (2008).

26. Kuehnbaum, N. L. \& Britz-McKibbin, P. New advances in separation science for metabolomics: Resolving chemical diversity in a post-genomic era. Chem. Rev. 113, 2437-2468 (2013).

27. Ellis, D. I. \& Goodacre, R. Metabolomics-assisted synthetic biology. Curr. Opin. Biotechnol. 23, 22-28 (2012).

28. Nagato, E. G. et al. ${ }^{1} \mathrm{H}$ NMR-based metabolomics investigation of Daphnia magna responses to sub-lethal exposure to arsenic, copper and lithium. Chemosphere 93 , 331-337 (2013).

29. Wu, H. et al. Proteomic and metabolomic responses of clam Ruditapes philippinarum to arsenic exposure under different salinities. Aquat. Toxicol. 136, 91-100 (2013).

30. Bagri, A. et al. Structural evolution during the reduction of chemically derived graphene oxide. Nat. Chem. 2, 581-587 (2010).

31. Zhang, Y. et al. Cytotoxicity effects of graphene and single-wall carbon nanotubes in neural phaeochromocytoma-derived PC12 cells. ACS Nano 4, 3181-3186 (2010).

32. Akhavan, O., Ghaderi, E. \& Akhavan, A. Size-dependent genotoxicity of graphene nanoplatelets in human stem cells. Biomaterials 33, 8017-8025 (2012).

33. Akhavan, O. Ghaderi, E. \& Esfandiar, A. Wrapping bacteria by graphene nanosheets for isolation from environment, reactivation by sonication, and inactivation by near-infrared irradiation. J. Phys. Chem. B 115, 6279-6288 (2011).

34. Hu, J., Wang, D., Forthaus, B. E. \& Wang, J. Quantifying the effect of nanoparticles on $\mathrm{As}(\mathrm{V})$ ecotoxicity exemplified by nano- $\mathrm{Fe}_{2} \mathrm{O}_{3}$ (magnetic) and nano- $\mathrm{Al}_{2} \mathrm{O}_{3}$. Environ. Toxicol. Chem. 31, 2870-2876 (2012).

35. Duarte, I. F. Following dynamic biological processes through NMR-based metabonomics: A new tool in nanomedicine? J. Control Release 153, 34-39 (2011).

36. Morsy, M. R., Jouve, L., Hausman, J. F., Hoffmann, L. \& Stewart, J. M. Alteration of oxidative and carbohydrate metabolism under abiotic stress in two rice (Oryza sativa L.) genotypes contrasting in chilling tolerance. J. Plant Physiol. 164, 157-67 (2007).

37. Marti, G. et al. Metabolomics reveals herbivore-induced metabolites of resistance and susceptibility in maize leaves and roots. Plant Cell Environ. 36, 621-639 (2013).

38. Hong, S.-Y. Roze, L. V. \& Linz, J. E. Oxidative stress-related transcription factors in the regulation of secondary metabolism. Toxins 5, 683-702 (2013).

39. Wang, C., Lu, J., Zhang, S., Wang, P., Hou, J. \& Qian, J. Effects of Pb stress on nutrient uptake and secondary metabolism in submerged macrophyte Vallisneria natans. Ecotox. Environ. Safe. 74, 1297-1303 (2011)

40. Mortimer, M., Kasemets, K., Vodovnik, M., Marinsek-Logar, R. \& Kahru, A. Exposure to $\mathrm{CuO}$ nanoparticles changes the fatty acid composition of Protozoa Tetrahymena thermophila. Environ. Sci. Technol. 45, 6617-6624 (2011).

41. Chen, R. et al. Differential uptake of carbon nanoparticles by plant and mammalian cells. Small 6, 612-617 (2010).

42. Lin, D. H. \& Xing, B. S. Root uptake and phytotoxicity of $\mathrm{ZnO}$ nanoparticles. Environ. Sci. Technol. 42, 5580-5585 (2008).
43. Wang, J. R. et al. Mechanisms of arsenic hyperaccumulation in Pteris vittata. Uptake kinetics, interactions with phosphate, and arsenic speciation. Plant Physiol. 130, 1552-1561 (2002).

44. Molin, M. et al. Humans seem to produce arsenobetaine and dimethylarsinate after a bolus dose of seafood. Environ. Res. 112, 28-39 (2012).

45. Watanabe, T. \& Hirano, S. Metabolism of arsenic and its toxicological relevance. Arch. Toxicol. 87, 969-979 (2013).

46. Duan, G. L., Zhu, Y. G., Tong, Y. P., Cai, C. \& Kneer, R. Characterization of arsenate reducatse in the extract of root and fronds of Chinese brake fern, an arsenic hyperaccumuator. Plant Physiol. 138, 461-469 (2005).

47. Cheng, L.-J. \& Cheng, T.-S. Oxidative effects and metabolic changes following exposure of greater duckweed (Spirodela polyrhiza) to diethyl phthalate. Aquat. Toxicol. 109, 166-175 (2012).

48. Hu, X. \& Zhou, Q. Novel hydrated graphene ribbon unexpectedly promotes aged seed germination and root differentiation. Sci. Rep. 4, 3782 (2014).

49. Nedjimi, B. \& Daoud, Y. Effects of calcium chloride on growth, membrane permeability and root hydraulic conductivity in two atriplex species grown at high (sodium chloride) salinity. J. Plant Nutr. 32, 1818-1830 (2009).

50. Davies, T. G. E. et al. Expression analysis of putative high-affinity phosphate transporters in Chinese winter wheats. Plant Cell Environ. 25, 1325-1339 (2002).

\section{Acknowledgments}

We thank Dr Yuming Chen, Mr Shaohu Ouyang, Dr Junjie Du and Ms Yuanyuan Gao for their help in the characteristics of GO nanosheets and cells. This work was financially supported by the National Natural Science Foundation of China (grant Nos: U1133006, 21037002 and 21307061), the Ministry of Education of China (grant No. IRT 13024),

Tianjin Natural Science Foundation (grant No. 14JCQNJC08900), the Doctoral Program of Higher Education of China (grant No. 2013003112016), the Postdoctoral Science Foundation of China (grant No. 2014M550138) and the Fundamental Research Funds for the Central Universities (grant no. 65121006).

\section{Author contributions}

H.X. and Z.Q. designed the research; H.X., M.L., Z.R., L.K. and K.J. performed the research; H.X. and M.L. analyzed the data; H.X. wrote the paper; and Z.Q. revised the manuscript.

\section{Additional information}

Supplementary information accompanies this paper at http://www.nature.com/ scientificreports

Competing financial interests: The authors declare no competing financial interests. How to cite this article: $\mathrm{Hu}$, X. et al. Graphene oxide amplifies the phytotoxicity of arsenic in wheat. Sci. Rep. 4, 6122; DOI:10.1038/srep06122 (2014).

(c) (i) (8) $\Theta$ This work is licensed under a Creative Commons Attribution-NonCommercialNoDerivs 4.0 International License. The images or other third party material in this article are included in the article's Creative Commons license, unless indicated otherwise in the credit line; if the material is not included under the Creative Commons license, users will need to obtain permission from the license holder in order to reproduce the material. To view a copy of this license, visit http:// creativecommons.org/licenses/by-nc-nd/4.0/ 